\section{Successful coronary scaffolding achieved with bioabsorbable stents}

The benefits of conventional stenting are primarily conferred within weeks of angioplasty, yet the subsequent endurance of the stent can lead to problems such as late stent thrombosis. In the PROGRESS-AMS trial, Erbel and colleagues assessed the feasibility of bioabsorbable coronary stents made from a magnesium alloydemonstrated in animal pilot studies to be completely absorbed within 2 months.

A total of 71 stents were successfully implanted in 63 consecutive patients (mean age 61.3 years) at eight centers. The stents were $10-15 \mathrm{~mm}$ long and $3.0-3.5 \mathrm{~mm}$ wide, and had mechanical characteristics similar to those of stainless steel equivalents.

Angiography after stenting demonstrated a mean reduction of diameter stenosis from $61.5 \%$ to $12.6 \%$, resulting in an acute lumen gain of $1.41 \mathrm{~mm}$. Four months later, diameter stenosis was $48.4 \%-a$ significant reduction compared with before the procedure. Intravascular ultrasound showed that the stent struts had been absorbed, but had left reflective markers of their former positions. No deaths, myocardial infarctions or incidences of stent thrombosis were recorded at 4, 6 or 12 months' follow-up. The overall rate of ischemia-driven target lesion revascularization was $23.8 \%$ at 4 months' and $26.7 \%$ at 12 months' follow-up.

Further development of absorbable stents is underway, and larger scale, randomized trials will be needed before these devices can be directly compared with conventional stents. These initial results suggest that implantation and absorption of these newer stents is safe, and that good short-term outcomes are possible.

Original article Erbel R et al. (2007) Temporary scaffolding of coronary arteries with bioabsorbable magnesium stents: a prospective, non-randomised multicentre trial. Lancet 369: 1869-1875

\section{Epoprostenol suitable for treatment of severe pulmonary hypertension in children}

The advent of epoprostenol for the treatment of pulmonary arterial hypertension has dramatically improved survival in adults; however, data on the use of this agent in pediatric cases are lacking. In a recent Heart paper, Lammers et al. review their experience of epoprostenol therapy in 39 children with severe pulmonary arterial hypertension. They conclude that epoprostenol is an effective and feasible treatment for this disorder even in young children.

All 39 children (aged 4 months to 17 years [median 5.4 years] at the onset of treatment) were in WHO functional class III or IV; 25 patients were being treated for idiopathic pulmonary arterial hypertension, and 14 patients for pulmonary arterial hypertension associated with congenital heart disease, connective tissue disease, chronic lung disease or HIV. All patients were severely symptomatic and underweight.

During a mean follow-up of 27 months, 7 patients died and 8 underwent transplantation. At 1, 2 and 3 years, the cumulative survival rates of all patients receiving epoprostenol were $94 \%, 90 \%$ and $84 \%$, respectively. During the first year of treatment, mean WHO functional class improved from 3.6 to $2.6(P<0.001)$, with an improvement noted in 30 of the 39 children. In addition, exercise capacity increased, as demonstrated by a mean $77 \mathrm{~m}$ improvement in 6 min walking distance in 28 children over a mean follow-up of 11.4 months $(P<0.003)$. The mean $z$ score for weight also improved, increasing from -1.55 to -1.16 during follow-up $(P<0.03)$.

Original article Lammers AE et al. (2007) Epoprostenol treatment in children with severe pulmonary hypertension. Heart 93: 739-743

\section{Rosiglitazone and the risk of myocardial infarction: interim analysis of the RECORD trial}

Following recent concern that the hypoglycemic agent rosiglitazone might increase the already elevated risk of myocardial infarction in patients with type 2 diabetes, Home et al. conducted an unplanned analysis of data from the ongoing Rosiglitazone Evaluated for Cardiac Outcomes and Regulation of Glycaemia in Diabetes (RECORD) trial.

The RECORD trial study population comprises 4,458 patients with type 2 diabetes. At baseline, those patients already taking a sulfonylurea were randomized to additionally receive either rosiglitazone $(n=1,103)$ or metformin $(n=1,122)$, while those patients taking metformin were 